\title{
Incidence and management of CAR-T neurotoxicity in patients with multiple myeloma treated with ciltacabtagene autoleucel in CARTITUDE studies
}

Adam D. Cohen (D ${ }^{1}$, Samir Parekh², Bianca D. Santomasso ${ }^{3}$, Jaime Gállego Pérez-Larraya (iD ${ }^{4}$, Niels W. C. J. van de Donk ${ }^{5}$, Bertrand Arnulf ${ }^{6}$, Maria-Victoria Mateos (iD ${ }^{7}$, Nikoletta Lendvai ${ }^{8}$, Carolyn C. Jackson ${ }^{8}$, Kevin C. De Braganca (iD ${ }^{8}$, Jordan M. Schecter ${ }^{8}$, Loreta Marquez $^{8}$, Erin Lee $\mathbb{D}^{8}{ }^{8}$, Ingrid Cornax ${ }^{8}$, Enrique Zudaire ${ }^{9}$, Claire Li $^{9}$, Yunsi Olyslager ${ }^{10}$, Deepu Madduri ${ }^{8}$, Helen Varsos ${ }^{8}$, Lida Pacaud ${ }^{11}$, Muhammad Akram ${ }^{11}$, Dong Geng ${ }^{11}$, Andrzej Jakubowiak (iD) ${ }^{12}$, Hermann Einsele (D) ${ }^{13}$ and Sundar Jagannath (iD ${ }^{2 \times}$

(c) The Author(s) 2022

Chimeric antigen receptor (CAR) T-cell therapies are highly effective for multiple myeloma (MM) but their impressive efficacy is associated with treatment-related neurotoxicities in some patients. In CARTITUDE-1, 5\% of patients with MM reported movement and neurocognitive treatment-emergent adverse events (MNTs) with ciltacabtagene autoleucel (cilta-cel), a B-cell maturation antigen-targeted CAR T-cell therapy. We assessed the associated factors for MNTs in CARTITUDE-1. Based on common features, patients who experienced MNTs were characterized by the presence of a combination of at least two variables: high tumor burden, grade $\geq 2$ cytokine release syndrome (CRS) or any grade immune effector cell-associated neurotoxicity syndrome (ICANS) after ciltacel infusion, and high CAR T-cell expansion/persistence. Strategies were implemented across the cilta-cel development program to monitor and manage patients with MNTs, including enhanced bridging therapy to reduce baseline tumor burden, early aggressive treatment of CRS and ICANS, handwriting assessments for early symptom detection, and extended monitoring/reporting time for neurotoxicity beyond 100 days post-infusion. After successful implementation of these strategies, the incidence of MNTs was reduced from $5 \%$ to $<1 \%$ across the cilta-cel program, supporting its favorable benefit-risk profile for treatment of MM.

Blood Cancer Journal (2022)12:32; https://doi.org/10.1038/s41408-022-00629-1

\section{INTRODUCTION}

Chimeric antigen receptor (CAR) T-cell therapies are novel and highly effective treatment approaches for various hematologic malignancies, including multiple myeloma (MM). The impressive remission rates with these therapies are sometimes accompanied by treatment-related neurotoxicities, which can be mild to life threatening [1-5]. Neurotoxicity events, including immune effector cell-associated neurotoxicity syndrome (ICANS), are heterogenous in nature with highly variable clinical presentation. Symptoms of ICANS may include aphasia, altered consciousness, cognitive skills impairment, motor weakness, seizures, and cerebral edema $[6,7]$. Other neurotoxicity events, usually with symptoms that do not fit the current definition for ICANS, have also been described with CAR T-cell therapies $[8,9]$.

The pathophysiology of neurotoxicities remains to be fully elucidated. ICANS can occur concurrently with cytokine release syndrome (CRS), a common side effect of CAR T-cell therapies, or days after resolution of CRS. The time to neurotoxicity onset after
CAR T-cell infusion also varies; some studies have reported an onset within 3-4 weeks of treatment $[7,10]$, whereas others have shown delayed events arising $>4$ weeks post treatment $[2,11]$. Thus, CAR T-cell-related neurotoxicities can have a highly variable course that requires careful monitoring and timely management to avoid potentially life-threatening or permanent neurologic sequelae.

Ciltacabtagene autoleucel (cilta-cel), a CAR T-cell therapy expressing two B-cell maturation antigen (BCMA)-targeting single binding domains, demonstrated deep and durable responses in heavily pretreated patients with relapsed/refractory $M M$ in the CARTITUDE- 1 phase $1 \mathrm{~b} / 2$ study $[12,13]$. Overall response rate was $98 \%$, with $80 \%$ of patients achieving stringent complete response [13]. After 18 months of follow-up, the progression-free survival and overall survival rates were $66 \%$ and $81 \%$, respectively. Similar to other CAR T-cell therapies [1-4, 9, 14, 15], cilta-cel-treated patients experienced ICANS. Here, we report the incidence of other CAR T-cell neurotoxicities characterized by movement and

\footnotetext{
${ }^{1}$ Abramson Cancer Center, University of Pennsylvania, Philadelphia, PA, USA. ${ }^{2}$ Mount Sinai Medical Center, New York, NY, USA. ${ }^{3}$ Department of Neurology, Memorial Sloan Kettering Cancer Center, New York, NY, USA. ${ }^{4}$ Department of Neurology, Clínica Universidad de Navarra, Pamplona, Spain. ${ }^{5}$ Department of Hematology, Amsterdam University Medical Center, Vrije Universiteit Amsterdam, Cancer Center Amsterdam, Amsterdam, The Netherlands. ${ }^{6}$ Hôpital Saint Louis, Paris, France. ${ }^{7}$ University Hospital of Salamanca/

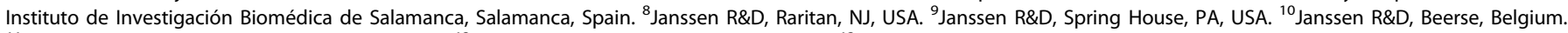
${ }^{11}$ Legend Biotech USA, Inc, Piscataway, NJ, USA. ${ }^{12}$ University of Chicago, Chicago, IL, USA. ${ }^{13}$ Universitätsklinikum Würzburg, Medizinische Klinik und Poliklinik II, Würzburg, Germany. Presented in part previously as congress abstracts at The American Society of Clinical Oncology Annual Meeting; Virtual format; June 4-8, 2021 (Abstract numbers 8005 and 8028). ${ }^{\times}$email: sundar.jagannath@mountsinai.org
} 
Table 1. Movement and neurocognitive treatment-emergent adverse events in CARTITUDE-1.

\begin{tabular}{ll} 
Category & Preferred term \\
Movement disorder & $\begin{array}{l}\text { Ataxia, Balance disorder, Bradykinesia, Cogwheel rigidity, Dysgraphia, Dyskinesia, Dysmetria, Essential tremor, Gait } \\
\text { disturbance, Hand-eye coordination impaired, Micrographia, Motor dysfunction, Myoclonus, Parkinsonism, Posture } \\
\text { abnormal, Resting tremor, Stereotypy, Tremor }\end{array}$ \\
\hline Cognitive impairment & $\begin{array}{l}\text { Amnesia, Apraxia, Bradyphrenia, Cognitive disorder, Confusional state, Depressed level of consciousness, Disturbance in } \\
\text { attention, Encephalopathy, Incoherent, Leukoencephalopathy, Loss of consciousness, Memory impairment, Mental } \\
\text { impairment, Mental status changes, Non-infective encephalitis, Psychomotor retardation }\end{array}$ \\
\hline Personality changes & Flat affect, Personality change, Reduced facial expression
\end{tabular}

neurocognitive treatment-emergent adverse events (MNTs) in CARTITUDE-1, which comprise a cluster of movement (e.g., micrographia, tremors), cognitive (e.g., memory loss, disturbance in attention), and personality changes (e.g. reduced facial expression, flat affect). Although some symptoms overlap with ICANS symptomatology (i.e., altered mental status, somnolence), MNT symptoms occur after a period of recovery from CRS and/or ICANS and may present in a unique pattern, including insidious onset; these symptoms are also generally non-responsive to steroids, often progressive and have longer duration than ICANS. Of note, patients also had normal to near normal ICE scores at the time of MNT presentation, which is inconsistent with the current literature definition of ICANS [6]. Possible associated factors, underlying pathology, patient management strategies, and implications for clinical practice are presented.

\section{METHODS \\ Study design and participants}

Patients were enrolled in the open-label phase 1b/2 CARTITUDE- 1 study (NCT03548207) in the USA (16 centers) and Japan (four centers). Full study design details and eligibility criteria have been previously described [12]. Briefly, eligible patients had a diagnosis of MM per International Myeloma Working Group criteria [16], received $\geq 3$ prior lines of therapy or were double refractory to a proteasome inhibitor and an immunomodulatory drug, and had received a proteasome inhibitor, an immunomodulatory drug, and an anti-CD38 antibody. Patients had to have documented disease progression $\leq 12$ months after the last line of therapy and were excluded if they had previously been treated with a CAR T-cell or BCMAtargeted therapy. Patients with known active or prior history of central nervous system involvement and those who exhibited clinical signs of meningeal involvement of MM were excluded from the study. If preexisting disease was suspected at screening, neurology consultation and dedicated baseline neuroimaging were considered.

\section{Procedures}

Apheresis was performed according to institutional standards, with a collection target of $6 \times 10^{9}$ peripheral blood mononuclear cells (range, $2-20 \times 10^{9}$ ). Bridging therapy was permitted when clinically indicated during the manufacturing process. Per protocol, patients could only receive shortterm bridging therapy with agents they had previously been exposed to and that had generated at least a response or stable disease. Cilta-cel was given as a single infusion on Day 1 (target dose $0.75 \times 10^{6}$ [range, $0.5-1.0 \times 10^{6}$ ] CAR+ viable $\mathrm{T}$ cells $/ \mathrm{kg}$ ) 5-7 days after the start of lymphodepletion (cyclophosphamide $300 \mathrm{mg} / \mathrm{m}^{2}$ and fludarabine $30 \mathrm{mg} / \mathrm{m}^{2}$ daily for 3 days).

This study was conducted in accordance with the Declaration of Helsinki and International Conference on Harmonization Good Clinical Practice guidelines. All patients provided informed consent. The study protocol, amendments, and relevant documents were approved by an independent ethics committee/institutional review board at each study center.

\section{Assessments}

Patients were monitored for safety and disease assessments from Day 1 to Day 100 post cilta-cel infusion and then every 28 days from Day 101 to study completion ( 2 years after the last patient was dosed). Survival status and subsequent anti-cancer therapy information were collected every 16 weeks following disease progression.
Adverse events were graded using National Cancer Institute Common Terminology Criteria for Adverse Events (NCl-CTCAE) version 5.0. In the combined analysis of CARTITUDE- 1 phase $1 \mathrm{~b}$ and phase 2, ICANS and CRS were graded by American Society for Transplantation and Cellular Therapy criteria [6], and other CAR T-cell neurotoxicities (onset after a period of recovery from CRS and ICANS) by NCI-CTCAE version 5.0.

In CARTITUDE-1, the cluster of symptoms that occurred with MNTs (a subset of other CAR T-cell neurotoxicities) were grouped into the following categories-movement disorder, cognitive impairment, and personality changes (Table 1). Patients were considered to have MNTs if they met all three of the following criteria: (i) must have reported at least one or more of the preferred terms in at least two of the above categories; (ii) these reported preferred terms must have occurred following the recovery of CRS and/or ICANS; and (iii) symptoms must have been assessed by the investigator as CAR T-cell-related neurotoxicity (but not recognized as ICANS).

\section{Potential associated factors for MNTs}

To determine the potential associated factors for MNTs, various parameters were evaluated including baseline demographics and disease characteristics; tumor burden; use of bridging therapies; viral infection prior to apheresis; prior radiotherapy of the brain; and selected baseline laboratory values of fibrinogen, C-reactive protein, ferritin, platelet counts, $\beta-2$ microglobulin, serum cytokines interleukin (IL)-6, IL-10, interferon (IFN)- $\gamma$, and serum soluble IL-2 receptor $a$, and estimated glomerular filtration rate.

Tumor burden is typically defined based on the degree of plasmacytosis; however, there is a lack of consensus on the definition. In this analysis, we defined tumor burden based not only on the degree of plasmacytosis but also the presence of paraprotein since bone marrow aspirates are subject to sampling error depending on the distribution of malignant plasma cells within the bone marrow space. Thus, patients were categorized as having high tumor burden at baseline (prior to lymphodepletion) if they had any of the following: plasma cell infiltrate in the bone marrow $\geq 80 \%$, serum M-spike $\geq 5 \mathrm{~g} / \mathrm{dL}$, or involved serum free light chain $\geq 5000 \mathrm{mg} / \mathrm{L}$. Those with low tumor burden had all of the following: plasma cell infiltrate in the bone marrow $<50 \%$, serum M-spike $<3 \mathrm{~g} / \mathrm{dL}$, and serum free light chain $<3000 \mathrm{mg} / \mathrm{L}$. Patients who did not fit either criterion were categorized as having intermediate tumor burden.

Parameters evaluated post cilta-cel infusion were: CRS and ICANS (by grade), supportive therapies for CRS and ICANS, CAR T-cell expansion and persistence (defined as peripheral blood CAR-T cells $C_{\max }$ of $>1000$ cells $/ \mu \mathrm{L}$ and CAR-T cells $>300$ cells/ $\mu \mathrm{L}$ at Day 56 ), hematology values (absolute lymphocyte counts $[A L C]$, absolute neutrophil counts, and platelet counts in the first 30 days), absolute CD4+ T-cell counts, absolute CAR+ T cells, frequency of CAR+ T cells, and cytokine levels (IL-6 and IFN- $\gamma$ ). Cerebrospinal fluid (CSF) samples of patients with MNTs were assessed for the presence of CAR+ T cells.

Chemistry, manufacturing, and controls assessment reviewed batches of the cilta-cel drug product used in patients with MNTs for any deviations that may have occurred during manufacturing.

\section{Monitoring and patient management strategies}

A safety management team evaluated the cases of MNTs in CARTITUDE-1 to identify and implement various changes in the conduct of ongoing studies across the CARTITUDE program. Amendments were made to all the global protocols and patient informed consent forms with the aim to provide guidance to investigators at study sites.

\section{Statistical analysis}

This analysis included the safety population from CARTITUDE-1 (all ciltacel-treated patients). Descriptive statistics and frequency distribution with 
CAR T-Cell Neurotoxicities

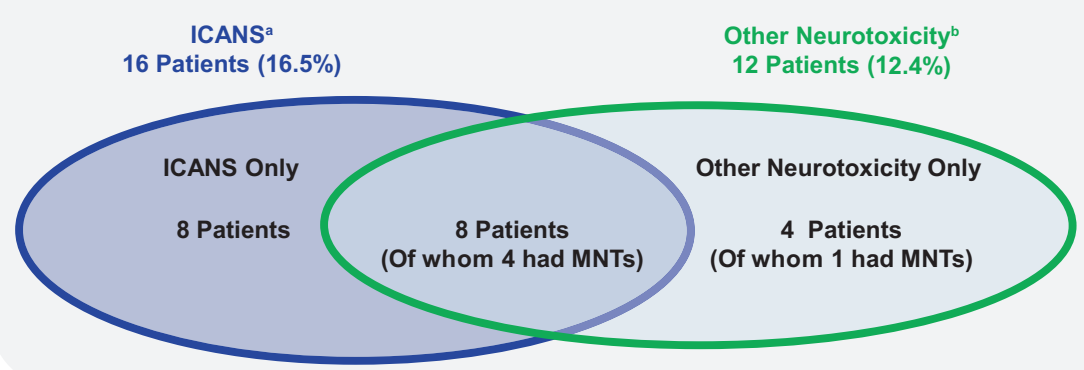

Fig. 1 Overview of CAR T-cell neurotoxicities in CARTITUDE-1. ${ }^{a}$ ICANS per ASTCT consensus criteria. ${ }^{b}$ Neurotoxicity as assessed by the investigator to be related to cilta-cel occurring after a period of recovery from CRS and/or ICANS. ICANS and other neurotoxicity events are not mutually exclusive; eight (8.2\%) patients experienced both ICANS and other neurotoxicity events of any grade. Patients in the otherneurotoxicity group $(n=12)$ include the subset with MNTs $(n=5)$. ASTCT American Society for Transplantation and Cellular Therapy, CAR chimeric antigen receptor, CRS cytokine release syndrome, ICANS immune effector cell-associated neurotoxicity syndrome, MNTs movement and neurocognitive treatment-emergent adverse events.

the numbers and percentages of patients in each category of the MNTs observed in CARTITUDE-1 were included as appropriate. Differences between patients with and without MNTs in the clinical variables were assessed using Wilcoxon rank sum test for continuous variables and Fisher's exact test for categorical variables. Odds ratios and confidence intervals for logistic regression, with MNTs as a dependent variable, were used to evaluate each of the clinical variables as an independent variable.

\section{RESULTS}

Neurotoxicity data from a total of 97 patients in CARTITUDE- 1 with a February 2021 cutoff date were included in this analysis, representing a median follow-up of 18 months. Detailed baseline characteristics of patients in CARTITUDE- 1 have been previously published [12]. Patients' median age was 61 years, 59\% were male, and $24 \%$ had high-risk cytogenetic profile-defined as the presence of del17p, $t(14: 16)$, and $t(4: 14)$. Patients had received a median of six prior lines of therapy; $88 \%$ were triple-class refractory, $42 \%$ were penta-drug refractory, and $99 \%$ were refractory to their last line of therapy.

Total CAR T-cell neurotoxicities, including ICANS and other CAR T-cell neurotoxicities, were observed in $20.6 \%$ of patients; nine $(9.3 \%)$ had grade $3 / 4$ events and one $(1.0 \%)$ had a grade 5 event. ICANS and other CAR T-cell neurotoxicities were not mutually exclusive as eight patients (8.2\%) experienced both (Fig. 1). ICANS occurred in $16(16 \%)$ patients, mostly grade $1 / 2(14 \%)$ with one patient each having a grade 3 and 4 event; 15 of the 16 patients had concurrent CRS. Median time to onset of ICANS was 8 days (range, 3-12 days) and the median duration was 4 days (range, 1-12). All patients had recovered from ICANS at data cutoff. Other CAR T-cell neurotoxicities occurred in $12(12.4 \%)$ patients; median onset was 26.5 days (range, 11-108) after cilta-cel infusion, and median time to resolution was 70 days (range, 2-159). Symptoms associated with other neurotoxicities were variable and wide ranging and have been previously described [12]. Of these 12 patients, five experienced MNTs as described below. Neurotoxicity in the remaining seven patients manifested as facial paralysis $(n=$ $1)$, neurotoxicity $(n=1)$, concentration impairment $(n=1)$, diplopia $(n=1)$, cranial nerve palsy $(n=1)$, sensory loss, ataxia, peripheral motor neuropathy, and peripheral sensory neuropathy (all in one patient), and altered mental status and nystagmus (both in one patient; Supplementary Table 1). Five of these 7 patients recovered from neurotoxicity, and 2 died of other causes.

\section{MNTs in CARTITUDE-1 and potential associated factors}

Five patients experienced MNTs, which included a cluster of movement, cognitive, and personality changes (Table 1). Details of these patients are described in Table 2 . One patient experienced a
Table 2. Characteristics of MNTs in CARTITUDE-1.

\begin{tabular}{|ll|}
\hline Characteristic & $\mathbf{N}=\mathbf{9 7}$ \\
\hline Patients with $\mathrm{MNT}^{\mathrm{a}}, n(\%)$ & $5(5.2)$ \\
\hline Maximum toxicity grade, $n(\%)$ & \\
\hline Grade 1 & 0 \\
\hline Grade 2 & $1(1.0)$ \\
\hline Grade 3 & $3(3.1)$ \\
\hline Grade 4 & 0 \\
\hline Grade 5 & $1(1.0)$ \\
\hline Median time to onset, days (range) & $27.0(14-108)$ \\
\hline Outcome of neurotoxic event, $\mathrm{n}(\%)$ & \\
\hline Recovered or resolved & 0 \\
\hline Not recovered or not resolved & $3(3.1)^{\mathrm{b}}$ \\
\hline Recovering or resolving & $1(1.0)$ \\
\hline Fatal & $1(1.0)$ \\
\hline
\end{tabular}

ICANS immune effector cell-associated neurotoxicity syndrome, MNTS movement and neurocognitive treatment-emergent adverse events.

${ }^{a}$ Events not reported as ICANS (i.e., onset after a period of recovery from cytokine release syndrome and ICANS).

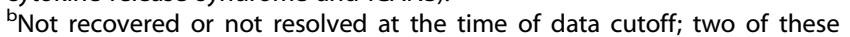
patients died due to other causes (one due to septic shock and one due to lung abscess).

grade 2 event, three patients experienced grade 3 events, and one patient experienced a grade 5 event; median time to onset was 27.0 days. The median onset of MNTs was 17 days (range, 3-94) after the recovery of CRS and ICANS. Supportive measures for MNTs included steroids, systemic chemotherapy (cyclophosphamide), intrathecal chemotherapy (methotrexate, cytarabine), anakinra, dasatinib, siltuximab, and other agents (e.g., carbidopa/ levodopa, levetiracetam) with limited or no observed improvement in symptoms. Three of the five patients with MNTs achieved stringent complete response and two achieved very good partial response, as assessed by an independent review committee, following treatment with cilta-cel.

Baseline and post-infusion characteristics in patients with and without MNTs are shown in Table 3. All five patients with MNTs were male and had received prior bridging therapy; three (60\%) had high tumor burden at baseline. Post cilta-cel infusion, all five patients developed grade $\geq 2$ CRS; four ( $80 \%$ ) had ICANS, and four (80\%) had high CAR T-cell expansion and persistence. Of the 92 patients without MNTs, $13(14.1 \%)$ had high tumor burden, 38 
Table 3. Baseline and post-infusion characteristics in patients with and without MNTs in CARTITUDE-1.

\begin{tabular}{|c|c|c|}
\hline \multirow[t]{2}{*}{ Baseline characteristic } & \multicolumn{2}{|l|}{ MNTs } \\
\hline & No $(n=92)$ & Yes $(n=5)$ \\
\hline \multicolumn{3}{|l|}{ Age, years; $n(\%)$} \\
\hline$<65$ & $59(64.1)$ & $3(60.0)$ \\
\hline $65-75$ & $26(28.3)$ & $1(20.0)$ \\
\hline$>75$ & $7(7.6)$ & $1(20.0)$ \\
\hline \multicolumn{3}{|l|}{ Sex, $n(\%)$} \\
\hline Female & $40(43.5)$ & 0 \\
\hline Male & $52(56.5)$ & $5(100.0)$ \\
\hline \multicolumn{3}{|l|}{ Race, $n$ (\%) } \\
\hline White & $64(69.6)$ & $5(100.0)$ \\
\hline African American & $17(18.5)$ & 0 \\
\hline Other & $11(12.0)$ & 0 \\
\hline \multicolumn{3}{|l|}{ Ethnicity, $n$ (\%) } \\
\hline Hispanic or Latino & $6(6.5)$ & 0 \\
\hline Non-Hispanic or Latino & $80(87.0)$ & $5(100.0)$ \\
\hline Not reported & $6(6.5)$ & 0 \\
\hline \multicolumn{3}{|l|}{ ECOG PS score ${ }^{\mathrm{a}}, n(\%)$} \\
\hline 0 & $36(39.1)$ & $3(60.0)$ \\
\hline 1 & $52(56.5)$ & $2(40.0)$ \\
\hline 2 & $4(4.3)$ & 0 \\
\hline \multicolumn{3}{|c|}{ Tumor burden category ${ }^{\mathrm{b}}, n(\%)$} \\
\hline High & $13(14.1)$ & $3(60.0)$ \\
\hline Intermediate & $21(22.8)$ & $1(20.0)$ \\
\hline Low & $58(63.0)$ & $1(20.0)$ \\
\hline \multicolumn{3}{|l|}{ Type of myeloma, $n(\%)$} \\
\hline $\lg G$ & $55(59.8)$ & $2(40.0)$ \\
\hline Non-lgG & $37(40.2)$ & $3(60.0)$ \\
\hline \multicolumn{3}{|c|}{ Measurable disease type, $n(\%)$} \\
\hline $\begin{array}{l}\text { Serum only, serum } \\
\text { and urine }\end{array}$ & $51(55.4)$ & $4(80.0)$ \\
\hline $\begin{array}{l}\text { Urine only, FLC, not } \\
\text { evaluable }\end{array}$ & $41(44.6)$ & $1(20.0)^{c}$ \\
\hline \multicolumn{3}{|c|}{ Extramedullary plasmacytoma, $n(\%)$} \\
\hline No & $80(87.0)$ & $4(80.0)$ \\
\hline Yes & $12(13.0)$ & $1(20.0)$ \\
\hline \multicolumn{3}{|c|}{ Bone-based plasmacytoma, $n(\%)$} \\
\hline No & $87(94.6)$ & $4(80.0)$ \\
\hline Yes & $5(5.4)$ & $1(20.0)$ \\
\hline \multicolumn{3}{|c|}{ Number of prior lines of therapy, $n(\%)$} \\
\hline $3-5$ & $46(50.0)$ & $3(60.0)$ \\
\hline$\geq 6$ & $46(50.0)$ & $2(40.0)$ \\
\hline \multicolumn{3}{|c|}{ Prior radiotherapy including brain area, $n$ (\%) } \\
\hline No & $84(91.3)$ & $5(100.0)$ \\
\hline Yes & $8(8.7)$ & 0 \\
\hline \multicolumn{3}{|l|}{ Prior bridging therapy, $n(\%)$} \\
\hline No & $24(26.1)$ & 0 \\
\hline Yes & $68(73.9)$ & $5(100.0)$ \\
\hline \multicolumn{3}{|c|}{ Type of prior bridging therapy, $n(\%)$} \\
\hline \multicolumn{3}{|l|}{ Daratumumab } \\
\hline No & $78(84.8)$ & $4(80.0)$ \\
\hline Yes & $14(15.2)$ & $1(20.0)$ \\
\hline
\end{tabular}

\begin{tabular}{|c|c|c|}
\hline \multirow[t]{2}{*}{ Baseline characteristic } & \multicolumn{2}{|l|}{ MNTs } \\
\hline & No $(n=92)$ & Yes $(n=5)$ \\
\hline \multicolumn{3}{|l|}{ Lenalidomide } \\
\hline No & $86(93.5)$ & $5(100.0)$ \\
\hline Yes & $6(6.5)$ & 0 \\
\hline \multicolumn{3}{|c|}{ Post-infusion characteristic } \\
\hline \multicolumn{3}{|c|}{ Total CAR + viable T-cells infused $\left(\times 10^{6}\right), n(\%)$} \\
\hline$<$ median value & $46(50.0)$ & $2(40.0)$ \\
\hline$\geq$ median value & $46(50.0)$ & $3(60.0)$ \\
\hline \multicolumn{3}{|c|}{ High cell expansion/persistence ${ }^{d}, n(\%)$} \\
\hline No & $84(91.3)$ & $1(20.0)$ \\
\hline Yes & $8(8.7)$ & $4(80.0)$ \\
\hline \multicolumn{3}{|l|}{ CRS, $n(\%)$} \\
\hline No & $5(5.4)$ & 0 \\
\hline Yes & $87(94.6)$ & $5(100.0)$ \\
\hline \multicolumn{3}{|c|}{ CRS maximum toxicity grade, $n(\%)$} \\
\hline Grade $<2$ & $54(58.7)$ & 0 \\
\hline Grade $\geq 2$ & $38(41.3)$ & $5(100.0)$ \\
\hline \multicolumn{3}{|l|}{ ICANS, $n(\%)$} \\
\hline No & $80(87.0)$ & $1(20.0)$ \\
\hline Yes & $12(13.0)$ & $4(80.0)$ \\
\hline
\end{tabular}

$C A R$ chimeric antigen receptor, $C_{\max }$ maximum $C A R$ transgene systemic level, CRS cytokine release syndrome, ECOG PS Eastern Cooperative Oncology Group performance status, FLC free light chain, ICANS immune effector cell-associated neurotoxicity syndrome, $I g G$ immunoglobulin $G$, MNTs movement and neurocognitive treatment-emergent adverse events. ${ }^{a}$ The last non-missing ECOG PS score on or prior to date of cilta-cel infusion was used; all patients met the inclusion criteria of ECOG PS score of 0 or 1 during screening.

${ }^{\mathrm{b}}$ Patients were categorized as having high tumor burden at baseline (prior to lymphodepletion) if they met any of the following: plasma cell infiltrate in the bone marrow $\geq 80 \%$, serum M-spike $\geq 5 \mathrm{~g} / \mathrm{dL}$, serum free light chain $\geq 5000 \mathrm{mg} / \mathrm{L}$. Those with low tumor burden had all of the following: plasma cell infiltrate in the bone marrow $<50 \%$, serum $\mathrm{M}$-spike $<3 \mathrm{~g} / \mathrm{dL}$, and serum free light chain $<3000 \mathrm{mg} / \mathrm{L}$. Patients who did not fit either criterion were categorized as having intermediate tumor burden.

'Patient was FLC-evaluable.

${ }^{d}$ Patients with peripheral blood CAR T cells $C_{\max }$ of $>1000$ cells $/ \mu \mathrm{L}$ and CAR T cells $>300$ cells $/ \mu \mathrm{L}$ at Day 56 .

(41.3\%) had grade $\geq 2$ CRS, 12 (13.0\%) had ICANS, and eight (8.7\%) had high CAR T-cell expansion and persistence.

Analysis of clinical laboratory values showed higher ALC, absolute CD4+ T cells, and absolute CAR + T cells on Days 14, 21 , and 28 post cilta-cel infusion in patients with MNTs than in those without MNTs (Fig. 2A-C). From Day 14 to Day 100 post cilta-cel infusion, the frequency of CAR+ T cells was higher in patients with MNTs compared with those who had other neurotoxicities without MNTs and those who had neither (Fig. 2D). Similarly, peak levels of IL- 6 and IFN- $\gamma$ were higher in patients with MNTs than in the other groups following treatment with cilta-cel (Fig. 2E and F). Pharmacokinetic analysis of CAR transgene levels in peripheral blood of patients with MNTs showed high CAR T-cell peak expansion and a trend of delayed $T_{\max }$ and high overall exposure (expansion and persistence) (Fig. 3). CSF examinations in two patients with MNTs for whom CSF samples were available showed CAR+ T cells.

Logistic regression analysis of odds ratios for potential associated factors showed an association between the occurrence of MNTs and high tumor burden at baseline before the start of 
A.
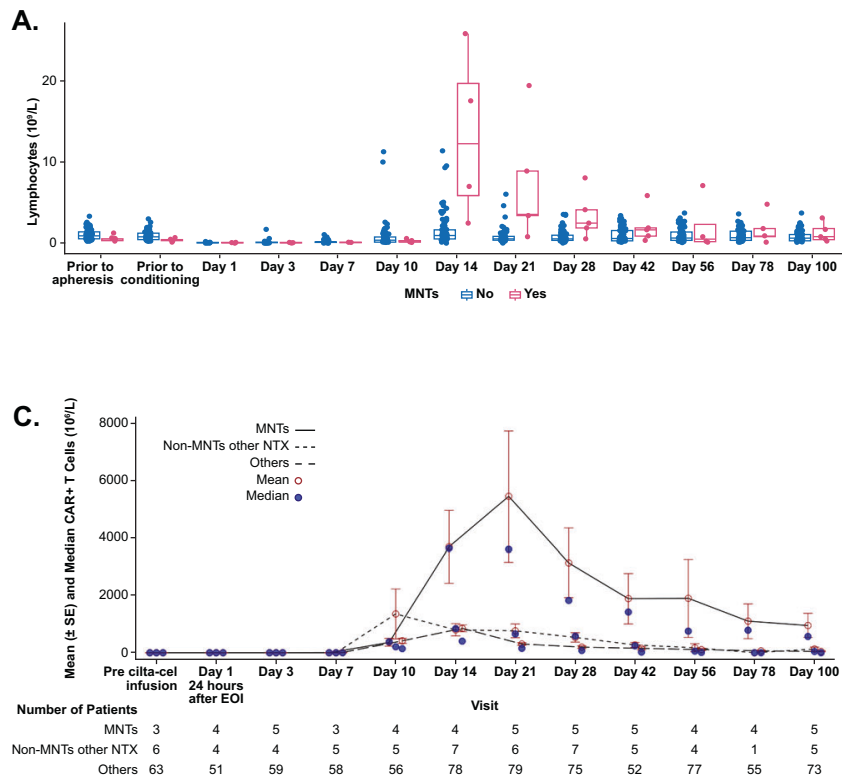

E.
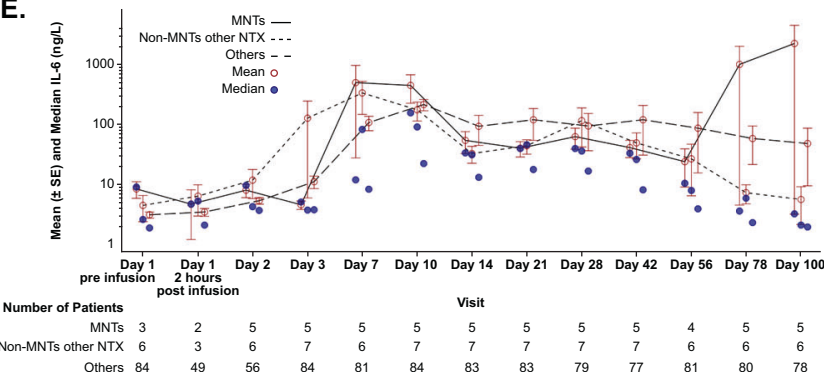

B.

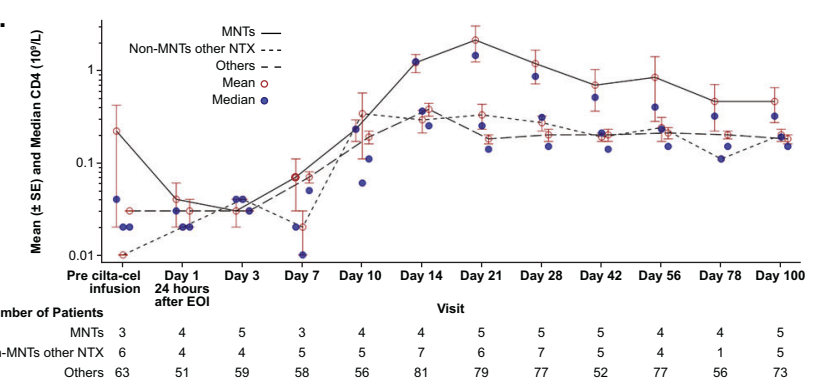

D.

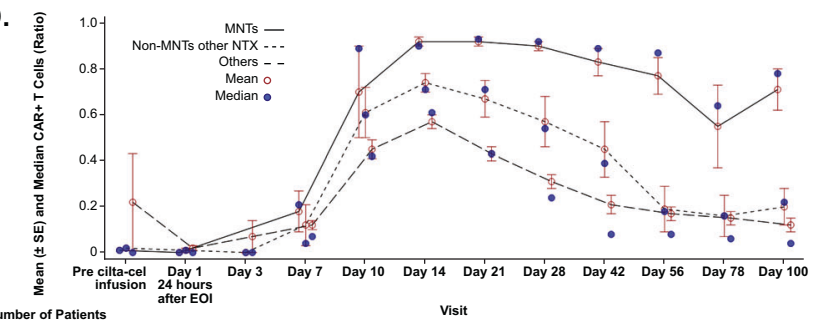

F.

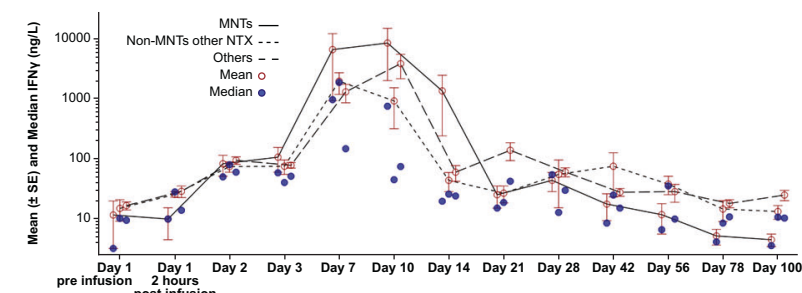

Number of Patients

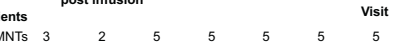

Fig. 2 Analysis of lymphocytes, $T$ cells, and cytokines over time following cilta-cel infusion in patients with and without MNTs in CARTITUDE-1. Values are shown for A lymphocyte counts; B absolute CD4+ T-cell counts; C absolute CAR+ T cells; D frequency of CAR+ T cells; E IL-6; and F IFN- $\gamma$. CAR chimeric antigen receptor, EOI end of infusion, IFN interferon, IL interleukin, MNT(s) movement and neurocognitive treatment-emergent adverse event(s), NTX neurotoxicity, SE standard error.

A.

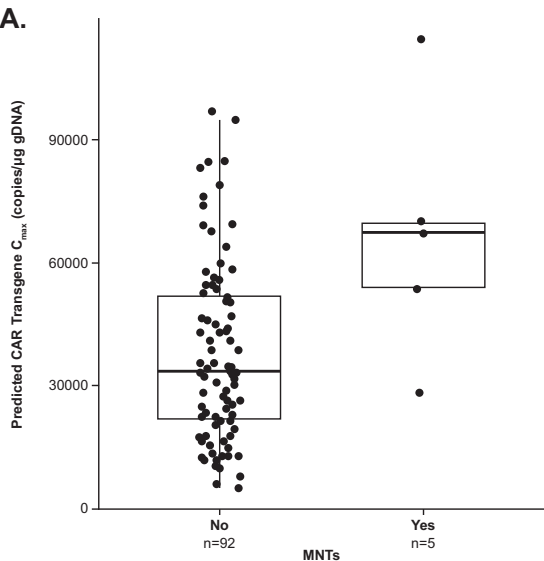

B.

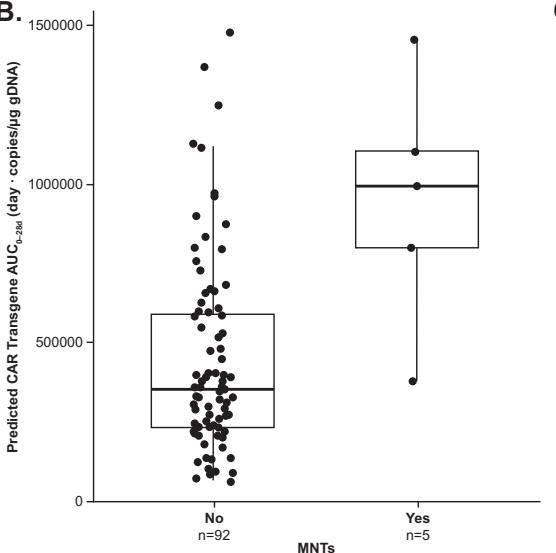

C.

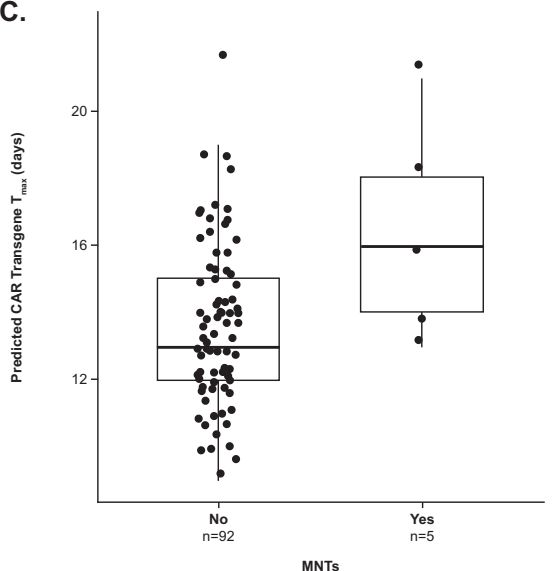

Fig. 3 Analysis of CAR transgene levels in patients with and without MNTs in CARTITUDE-1. Predicted values are shown for A $C_{\text {max }}$ B $A U C_{0-28 d}$, and $C T_{\text {max }} . A U C_{0-28 d}$ area under the CAR transgene systemic level-time curve from the first dose to Day 28, CAR chimeric antigen receptor, $C_{\max }$ maximum CAR transgene systemic level, gDNA genomic DNA, MNTs movement and neurocognitive treatment-emergent adverse events, $T_{\max }$ time of maximum cilta-cel transgene expansion.

lymphodepletion, high levels of IL- 6 at baseline, grade $\geq 2$ CRS, incidence of ICANS, high CAR T-cell expansion and persistence, and high ALC (including absolute CD4+ T cells) on Days 14, 21, and 28 post cilta-cel infusion (Fig. 4).

As noted above, a trend toward higher IL- 6 levels at baseline was seen in patients with MNTs versus those without, but there were no relevant differences in baseline levels of IL-10, IFN- $\gamma$, or IL2 receptor a between patients with and without MNTs (Supplementary Fig. 1). The CAR+ T-cell CD4/CD8 ratio was similar in patients with or without MNTs (Supplementary Fig. 2). Memory T-cell subsets at apheresis showed no clear differences among patients with ICANS, other CAR T-cell neurotoxicities, or MNTs 


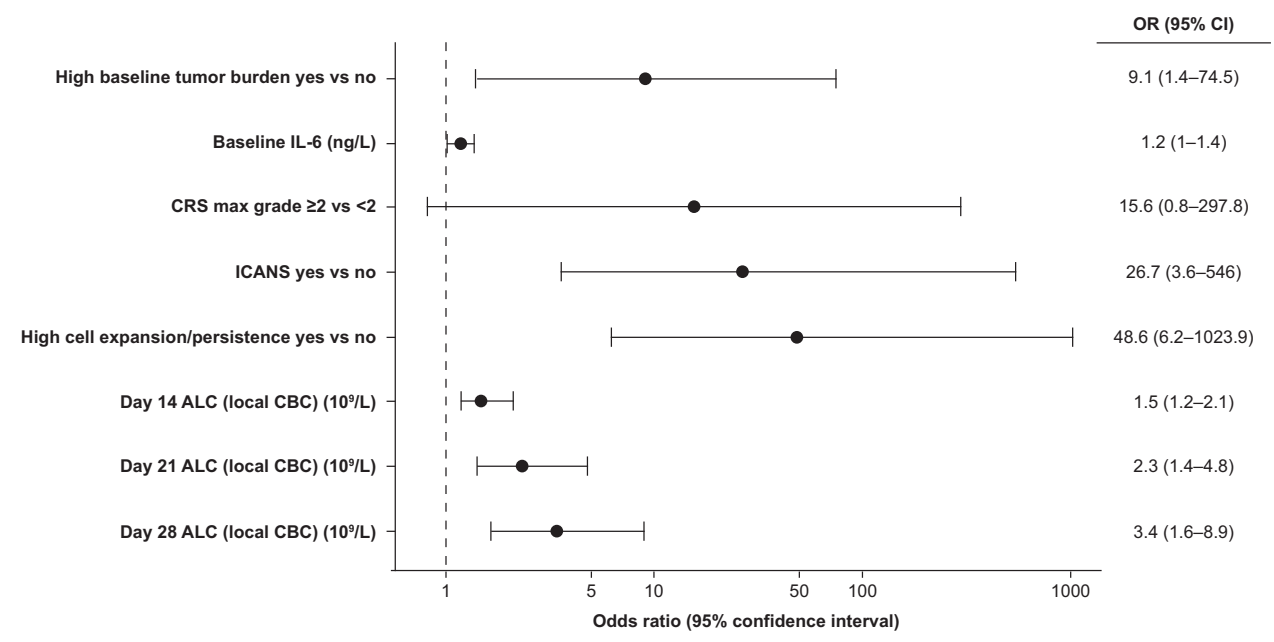

Fig. 4 Forest plot of potential factors associated with MNTs in CARTITUDE-1. ALC absolute lymphocyte count, CBC complete blood count, CRS cytokine release syndrome, ICANS immune effector cell-associated neurotoxicity syndrome, IL interleukin, MNTS movement and neurocognitive treatment-emergent adverse events.

(Supplementary Fig. 3). Similarly, treatment dose-response relationship showed no apparent trend between the infused cilta-cel dose $\left(0.75 \times 10^{6}\right.$ [range, $0.5-1.0 \times 10^{6}$ ] CAR+ viable T cells/ $\mathrm{kg}$ ) and other CAR T-cell neurotoxicities (Supplementary Fig. 4).

Chemistry, manufacturing, and controls assessment of the manufacturing process of CAR-T vector batches for the five patients with MNTs showed no relevant deviations in the manufacturing process. Furthermore, there were no notable product characteristics that would have distinguished patients who had MNTs from those who did not experience these events (data not shown).

Of the five patients with MNTs in CARTITUDE-1, one had ongoing symptoms at the time of data cutoff, one was recovering/ resolving, and three died. The causes of death were lung abscess, septic shock, and neurotoxicity on Days 119, 162, and 247 post cilta-cel infusion, respectively. All three fatal events were considered to be treatment-related by the investigator. Neuropathology findings from the two patients who had autopsy showed focal gliosis and T-cell infiltrate $(C D 8+>C D 4+)$ in the basal ganglia; it is unknown if these were CAR+ T cells. No abnormalities were reported in other brain regions (i.e., cerebellum, substantia nigra) potentially associated with MNTs, and there was preservation of pigmentation in the substantia nigra. In one of the two patients, while brain magnetic resonance imaging and head computed tomography scans did not demonstrate any significant abnormalities, FDG-PET scan showed hypometabolism in the basal ganglia. A dopamine uptake scan was negative, indicating normal dopamine signaling.

\section{Monitoring and patient-management strategies}

To minimize risk for MNTs, several preventive, monitoring, and patient management strategies were implemented across all ongoing studies in the cilta-cel clinical development program based on the results described above (Fig. 5). Preventive measures included fewer restrictions per protocol on the investigator's choice of bridging therapy used, including its duration, to reduce baseline tumor burden before cilta-cel infusion (i.e., enhanced bridging therapy based on the investigator's choice), and a risk-benefit discussion for patients with high baseline disease burden, particularly in those with progressive disease despite bridging therapy. Monitoring strategies included handwriting assessments using a novel handwriting tool for early detection of neurotoxicity symptoms (Supplementary Table 2), and extended monitoring and reporting time for CAR T-cell neurotoxicities up to
1 year after cilta-cel infusion. Management strategies included early and aggressive supportive care including steroids for anygrade ICANS, especially in patients with high tumor burden, and tocilizumab for any-grade ICANS with concurrent CRS. Guidance for physicians on management included potential diagnostics for new neurologic or psychiatric symptoms (see Supplementary Table 3).

\section{MNTs in ongoing trials with cilta-cel}

More than 150 patients were subsequently dosed across the ciltacel clinical development program since the implementation of monitoring and patient management strategies as described above.

As of the April 15, 2021 data cutoff, one patient in the multicohort phase 2 CARTITUDE-2 study (NCT04133636) developed other neurotoxicity, presenting with a cluster of symptoms characteristic of MNTs. As previously described, CARTITUDE-2 is evaluating cilta-cel safety and efficacy in various clinical settings for patients with MM [17]. This patient (male; 44 years of age), who was enrolled in cohort B (one prior line of therapy, early relapse), had characteristics that were identified among patients in CARTITUDE-1 as associated factors for MNTs such as high tumor burden at baseline, worsening disease burden despite bridging therapy, development of grade 4 CRS, and high CAR T-cell expansion and persistence. ALC on Day 21 post cilta-cel infusion was $8.4 \times 10^{9} / \mathrm{L} ; \mathrm{CD} 4+\mathrm{T}$-cell count on Day 14 was not available for this patient. Micrographia was one of the first neurologic manifestations of MNTs in this patient. The patient also developed variable and wide-ranging symptoms associated with these neurotoxicity events and had a cluster of MNTs including bradykinesia, rigidity, bradyphrenia, flat effect, apathy, gait disorder, and cognitive impairment which began on Day 38 after cilta-cel infusion. After treatment with high-dose methylprednisolone, plasmapheresis, and intravenous immunoglobulin, the patient was reported to be stable with mild improvement at data cutoff, and had achieved very good partial response as best response to treatment with cilta-cel.

\section{DISCUSSION}

A single infusion of cilta-cel resulted in unprecedented efficacy in heavily pretreated patients with MM in the CARTITUDE- 1 study [12]. Responses were early, durable, and deepened over time, and the highest (overall response rate, 98\%; stringent complete 


\section{Preventative strategies}

- Enhanced bridging therapy ${ }^{a}$ to reduce baseline tumor burden (may have included therapies to which a patient was not previously exposed)

- Performing neuroimaging (e.g., magnetic resonance imaging and electroencephalogram) at screening and/or neurology consultation in patients with preexisting neurologic disease

- Risk-benefit discussion prior to cilta-cel treatment for patients with large baseline disease burden, particularly those with progressive disease despite bridging therapy

- Use of prophylactic antimicrobials up to 6 months or longer after CAR T-cell infusion per institutional guidelines or consistent with post-ASCT consensus

Monitoring strategies
- Consultation and evaluation at the first sign of neurotoxicity, including CAR T-cell-related
neurotoxicity (e.g., ICANS) and raised intracranial pressure/cerebral edema
- Hospitilization for grade $\geq 2$ CAR T-cell-related neurotoxicity (e.g., ICANS) temporally associated
with CRS
- Neurologic evaluation with new onset of headache, convulsions, speech disorders, visual
disorders; disturbances in consciousness, confusion and disorientation, and coordination;
balance disorders, mental status changes, movement disorders, cognitive impairments,
personality changes
- Evaluation of infectious (e.g., human herpes virus), autoimmune, or paraneoplastic and
tumoral or metabolic etiologies at first sign of neurotoxicity in blood, cerebro spinal fluid,
and/or radiologic imaging
Performing immune effector cell-associated encephalopathy assessment tool at
baseline and at least daily after first symptoms of CAR T-cell neurotoxicities (e.g., ICANS or
other neurotoxicities) are suspected and until resolution
- Adding routine monitoring with regular handwriting assessments for early detection of
micrographia, dysgraphia, or agraphia
- Extending monitoring and reporting time for CAR T-cell neurotoxicity beyond the 100-day
period post cilta-cel infusion

\section{Management strategies}

- Early and more aggressive supportive care (including steroids) for any-grade ICANS, especially in patients with high tumor burden

- Consider administration of tocilizumab for any grade of ICANS with concurrent CRS, and/or dexamethasone (grade 1-3) or methylprednisolone (grade 4)

- Use of other cytokine-targeting therapies (e.g., anti-IL-1) based on institutional practice, especially for cases of neurotoxicity that do not respond to tocilizumab and corticosteroids

- Consider non sedating, anti seizure medicines (e.g., levetiracetam) for seizure prophylaxis for any grade 2 or higher neurologic toxicities

Fig. 5 Patient-management strategies for neurologic adverse events following treatment with cilta-cel. ${ }^{\mathrm{a}}$ Fewer restrictions (per protocol) on the investigator's choice of bridging therapy, including its duration, to reduce baseline tumor burden before cilta-cel infusion. ASTCT American Society for Transplantation and Cellular Therapy, CAR chimeric antigen receptor, CRS cytokine release syndrome, ICANS immune effector cell-associated neurotoxicity syndrome, IL interleukin.

response, $80 \%$ ) achieved to date in this patient population $[12,13]$. Responses were maintained in an estimated $66 \%$ of patients with no disease progression or death for 18 months after cilta-cel administration. Considerations of the benefits of cilta-cel treatment must also be balanced by potential risks. CAR T-cellrelated neurotoxicities, including ICANS and other CAR T-cell neurotoxicities, occurred in $20(21 \%)$ cilta-cel-treated patients in CARTITUDE-1. Five (5\%) of these patients presented with neurotoxicities beyond ICANS that were characterized by a cluster of MNTs. Symptoms were wide-ranging, included movement, cognitive, and personality changes, and occurred after a period of recovery from CRS and/or ICANS. All five patients had grade $\geq 2$ CRS and most had ICANS post cilta-cel infusion. Based on common features among these patients, potential associated factors were identified: a combination of at least two variables such as high tumor burden, grade $\geq 2$ CRS post-infusion, ICANS post-infusion, and high CAR T-cell expansion and persistence. Other associated factors included high baseline IL-6, high lymphocyte counts on Days 14,21 , and 28 post-infusion, and high peak levels of IL- 6 and IFN- $\gamma$ in peripheral blood. There were no relevant deviations in the manufacturing process of the CAR-T vector batches for the five patients with MNTs.
Similar to other CAR T-cell therapies [1-4, 9, 14, 15], ciltacel-treated patients experienced ICANS. As of June 30, 2021, a total of 1374 cases of neurotoxicity, including ICANS, were recorded in the US Food and Drug Administration Adverse Event Reporting System for the three approved anti-CD19 CAR T-cell therapies for hematologic cancers [5]. These neurotoxicities had a wide range of symptoms, with varying time to onset and duration, including headache, encephalopathy, tremor, confusional state, delirium, agitation, somnolence, sleep disorders, peripheral neuropathy, parkinsonism, rigidity/cogwheeling, and ataxia [2$4,7,9,14,15,18]$. Neurotoxicities were observed with BCMAtargeting anti-myeloma CAR T-cell therapies at rates ranging from $13 \%$ to $44 \%[1,8,19-21]$. Additionally, symptoms characteristic of MNTs (i.e., grade 3 parkinsonism) were reported with the approved anti-BCMA CAR-T therapy, idecabtagene vicleucel, in another study in MM [20]. Although MNTs are numerically higher in CARTITUDE-1, similarities in the type of neurologic events across various CAR T-cell therapies indicate this phenomenon may be attributable to a class effect. We investigated BCMA expression in normal brain as a potential explanation for this class effect but found no detectable immunoreactivity to BCMA protein in the brain samples of normal adults [22], which precluded any 
determination of a relationship between BCMA expression and incidence of MNTs but does not preclude aberrant expression in selected areas of the brain.

The underlying pathophysiology of neurotoxicities with CAR T-cell therapies is poorly understood. Reports in the literature suggest markers for neurotoxicity associated with BCMA-directed CAR T-cell therapies are similar to those with CD19-directed immunotherapies, and have demonstrated a correlation of ICANS with the presence and severity of CRS, and high tumor burden $[7,8,10,23,24]$. In one study of patients treated with CD19directed CAR T-cell therapy, CSF levels of S100 calcium-binding protein $B$ and glial fibrillary acidic protein increased during ICANS, indicating astrocyte injury [25]. Other studies have proposed that increases in the levels of IL-6, IFN- $\gamma$, and TNF- $a$ from CAR T cells may induce endothelial activation, which may in turn disrupt the blood-brain barrier and lead to development of ICANS [7, 10]. Although the clinical presentation of MNTs overlaps with Parkinson's disease, neuropathology findings in the two patients with MNTs in CARTITUDE-1 for whom autopsies were available showed intact substantia nigra, suggesting a distinct pathophysiology of these events. These autopsy findings were consistent with a negative dopamine uptake scan in one patient and lack of response to treatment with carbidopa/levodopa in both patients, providing further evidence of a pathophysiology that is distinct from Parkinson's disease.

In the absence of well-documented predictors of neurotoxicities in the literature, vigilant monitoring is recommended for prospective patients treated with CAR T-cell therapies, and is critical for early detection, adequate management, and minimization of morbidity. This was the rationale for the development and implementation of patient management strategies to minimize the incidence of CAR T-cell neurotoxicities during the CARTITUDE clinical trials. Guidance for physicians includes recommendations for enhanced bridging therapy to reduce baseline tumor burden, early aggressive treatment of CRS and ICANS, handwriting assessments for early detection of neurotoxicity symptoms, and extended monitoring and reporting time for CAR T-cell neurotoxicity beyond 100 days post-cilta-cel infusion. The success of monitoring and patient management strategies was demonstrated by the reduced incidence of MNTs after their implementation in the cilta-cel program as of the cutoff date for this report. With over 150 patients subsequently dosed across the cilta-cel clinical development program, the overall incidence of MNTs decreased from $5 \%$ to $<1 \%$.

While high CAR T-cell expansion, persistence, or frequency have emerged as potential factors associated with MNTs, the utility of these parameters in real-world clinical settings is limited. CAR-T levels are often difficult to measure, and symptoms of MNTs may manifest in the patient before any evidence of prolonged CAR T-cell persistence. In our analysis, elevated levels of CD4 $+\mathrm{T}$ cells $\left(>1000 \times 10^{6} / L\right)$ on Day 14 post-infusion, and higher ALC ( $>3 \times$ $\left.10^{9} / \mathrm{L}\right)$ on $\sim$ Day 21 post-infusion appear to correlate with a higher risk of developing MNTs, particularly in men. Hence, these biomarkers (independent of each other) may be useful in routine clinical practice as they can be assessed rapidly and before prolonged CAR T-cell persistence is detected. As expected, flow cytometry analysis of lymphocytes between Day 14 and Day 28 post-infusion showed a T-cell population primarily composed of CAR+ T cells. This suggests a potential for the utility of CD4+ $T$ cells on Day 14 and ALC on Day 21 post-infusion as surrogate markers for CAR-T levels. However, our findings show not all patients with $\mathrm{ALC}>3 \times 10^{9} / \mathrm{L}$ on Day 21 post-infusion develop MNTs. Therefore, this hypothesis warrants further validation in a larger cohort given that in over 150 patients subsequently treated with cilta-cel, only one developed MNTs. Notably, this patient had an ALC of $8.4 \times 10^{9} / \mathrm{L}$ on Day 21 post-infusion; CD4+ T-cell count on Day 14 post-infusion was not available.
Limitations of our study should be noted. In CARTITUDE-1, the number of patients with MNTs was small; hence further analysis of associated factors for these toxicities is needed. As of the cutoff date for this report, the CARTITUDE-2 study is enrolling, and patient follow-up is ongoing; subsequent analyses of patients in various cohorts of this multicohort study will inform the safety profile of cilta-cel. Cilta-cel is being evaluated in earlier line settings in the phase 3 studies of CARTITUDE-4 (NCT04181827) and CARTITUDE-5 (NCT04923893), which will further inform the risk factors for CAR T-cell neurotoxicities, including MNTs.

Patients completing CARTITUDE- 1 and other cilta-cel clinical trials will be enrolled in a long-term follow-up study for continued monitoring for up to 15 years. A planned observational postauthorization safety study will focus on areas including characterization and evaluation of identified risks, and long-term safety. Thus, the knowledge base about cilta-cel treatment of MM will continue to grow as data emerge on longer term efficacy and safety, mechanism of action, and patient perspectives.

\section{DATA AVAILABILITY}

The data sharing policy of Janssen Pharmaceutical Companies of Johnson \& Johnson is available at https://www.janssen.com/clinical-trials/transparency. As noted on this site, requests for access to the study data can be submitted through Yale Open Data Access (YODA) Project site at http://yoda.yale.edu.

\section{REFERENCES}

1. Munshi NC, Anderson LD Jr, Shah N, Madduri D, Berdeja J, Lonial S, et al. Idecabtagene vicleucel in relapsed and refractory multiple myeloma. $\mathrm{N}$ Engl J Med. 2021;384:705-16.

2. Schuster SJ, Bishop MR, Tam CS, Waller EK, Borchmann P, McGuirk JP, et al. Tisagenlecleucel in adult relapsed or refractory diffuse large B-cell lymphoma. $\mathrm{N}$ Engl J Med. 2019;380:45-56.

3. Wang M, Munoz J, Goy A, Locke FL, Jacobson CA, Hill BT, et al. KTE-X19 CAR T-cell therapy in relapsed or refractory mantle-cell lymphoma. $N$ Engl J Med. 2020;382:1331-42.

4. Neelapu SS, Locke FL, Bartlett NL, Lekakis LJ, Miklos DB, Jacobson CA, et al. Axicabtagene ciloleucel CAR T-cell therapy in refractory large B-cell lymphoma. $\mathrm{N}$ Engl J Med. 2017;377:2531-44.

5. FDA Adverse event reporting system public dashboard. 2020. https://fis.fda.gov.

6. Lee DW, Santomasso BD, Locke FL, Ghobadi A, Turtle CJ, Brudno JN, et al. ASTCT consensus grading for cytokine release syndrome and neurologic toxicity associated with immune effector cells. Biol Blood Marrow Transpl. 2019;25:625-38.

7. Santomasso BD, Park JH, Salloum D, Riviere I, Flynn J, Mead E, et al. Clinical and biological correlates of neurotoxicity associated with CAR T-cell therapy in patients with B-cell acute lymphoblastic leukemia. Cancer Discov. 2018;8:958-71.

8. Raje N, Berdeja J, Lin Y, Siegel D, Jagannath $S$, Madduri D, et al. Anti-BCMA CAR T-cell therapy bb2121 in relapsed or refractory multiple myeloma. N Engl J Med. 2019;380:1726-37.

9. Kymriah. Prescribing information. Novartis Pharmaceuticals Corporation; 2021.

10. Gust J, Hay KA, Hanafi LA, Li D, Myerson D, Gonzalez-Cuyar LF, et al. Endothelial activation and blood-brain barrier disruption in neurotoxicity after adoptive immunotherapy with CD19 CAR-T cells. Cancer Discov. 2017;7:1404-19.

11. Neelapu SS, Tummala S, Kebriaei P, Wierda W, Gutierrez C, Locke FL, et al. Chimeric antigen receptor T-cell therapy-assessment and management of toxicities. Nat Rev Clin Oncol. 2018;15:47-62.

12. Berdeja JG, Madduri D, Usmani SZ, Jakubowiak A, Agha M, Cohen AD, et al. Ciltacabtagene autoleucel, a B-cell maturation antigen-directed chimeric antigen receptor T-cell therapy in patients with relapsed or refractory multiple myeloma (CARTITUDE-1): a phase 1b/2 open-label study. Lancet 2021;398:314-24.

13. Usmani SZ, Berdeja JG, Madduri D, Jakubowiak AJ, Agha ME, Cohen AD. et al. Ciltacabtagene autoleucel, a B-cell maturation antigen (BCMA)-directed chimeric antigen receptor T-cell (CAR-T) therapy, in relapsed/refractory multiple myeloma (R/R MM): Updated results from CARTITUDE-1. J Clin Oncol. 2021;39 (15_suppl):8005

14. Yescarta. Prescribing information. Kite Pharma; 2021.

15. Tecartus. Prescribing information. Kite Pharma; 2021.

16. Rajkumar SV, Harousseau JL, Durie B, Anderson KC, Dimopoulos M, Kyle R, et al. Consensus recommendations for the uniform reporting of clinical trials: report of the International Myeloma Workshop Consensus Panel 1. Blood 2011;117:4691-5. 
17. Einsele H, van de Donk NWCJ, Arnulf B, Schecter JM, De Braganca KC, Varsos $\mathrm{H}_{\text {, }}$ et al. CARTITUDE-2: Phase 2 multicohort study of ciltacabtagene autoleucel, a B-cell maturation antigen-directed chimeric antigen receptor T-cell therapy, in patients with multiple myeloma. Transpl Cell Ther. 2021;27:S433-42021.

18. Maude SL, Laetsch TW, Buechner J, Rives S, Boyer M, Bittencourt H, et al. Tisagenlecleucel in children and young adults with B-cell lymphoblastic leukemia. $\mathrm{N}$. Engl J Med. 2018;378:439-48.

19. Mailankody S, Jakubowiak AJ, Htut M, Costa LJ, Lee K, Ganguly S, et al. Orvacabtagene autoleucel (orva-cel), a B-cell maturation antigen (BCMA)-directed CAR $\mathrm{T}$ cell therapy for patients (pts) with relapsed/refractory multiple myeloma (RRMM): update of the phase 1/2 EVOLVE study (NCT03430011). J Clin Oncol. 2020;38:8504. 15_suppl

20. ABECMA. Prescribing information. Celgene Corporation, a Bristol-Myers Squibb Company; 2021

21. Lin Y, Raje NS, Berdeja JG, Siegel DS, Jagannath S, Madduri D. et al. Idecabtagene vicleucel (ide-cel, bb2121), a BCMA-directed CAR T cell therapy, in patients with relapsed and refractory multiple myeloma: updated results from phase 1 CRB-401 study. Blood . 2020;136(Suppl 1):26-27.

22. Marella M, Yao X, Carreira V, Bustamante MF, Clark HB, Jackson CC, et al. Comprehensive $\mathrm{BCMA}$ expression profiling in adult normal human brain suggests a low risk of on-target neurotoxicity in BCMA-targeting multiple myeloma therapy. J Histochem Cytochem. 2022 [in press].

23. Cohen AD, Garfall AL, Stadtmauer EA, Melenhorst JJ, Lacey SF, Lancaster E, et al. B cell maturation antigen-specific CAR $T$ cells are clinically active in multiple myeloma. J Clin Investig. 2019;129:2210-21.

24. Mailankody S, Htut M, Lee KP, Bensinger W, Devries T, Piasecki J. et al. JCARH125, anti-BCMA CAR T-cell therapy for relapsed/refractory multiple myeloma: Initial proof of concept results from a phase $1 / 2$ multicenter study (EVOLVE). Blood . 2018;132(Suppl 1):957

25. Gust J, Finney OC, Li D, Brakke HM, Hicks RM, Futrell RB, et al. Glial injury in neurotoxicity after pediatric CD19-directed chimeric antigen receptor $\mathrm{T}$ cell therapy. Ann Neurol. 2019;86:42-54

\section{ACKNOWLEDGEMENTS}

This study was funded by Janssen Research \& Development, LLC and Legend Biotech, Inc. Medical writing support was provided by Jaya Kolipaka of Eloquent Scientific Solutions, and funded by Janssen Global Services, LLC. The authors thank the patients who participated in the study and their families and caregivers, the physicians and nurses who cared for patients and supported this clinical trial, the staff members at the study sites, and the staff members involved in data collection and analyses.

\section{AUTHOR CONTRIBUTIONS}

AC, SJ, SP, BS, JGP-L, NWCJvdD, BA, M-VM, AJ, and HE contributed to study design, conduct, data analysis and interpretation. NL, CCJ, KCDB, JMS, LM, EL, IC, EZ, CL, YO, $D M, H V, L P, M A$, and DG contributed to the study design, conduct, data acquisition, and data analysis and interpretation. All authors participated in drafting and revising the manuscript, and approved the final version for submission. The corresponding author had full access to all the data in the study and had final responsibility for the decision to submit for publication.

\section{COMPETING INTERESTS}

AC served in a consulting or advisory role for AstraZeneca, Bristol-Myers Squibb, Celgene, GlaxoSmithKline, Genentech/Roche, Janssen, and Takeda, and received patents, royalties, and other intellectual property from Novartis. SJ is a consultant for Bristol Myers Squibb, Janssen, Karyopharm Therapeutics, Merck, Sanofi, and Takeda Pharmaceuticals. SP is supported by the National Cancer Institute (NCl) (R01
CA244899, CA252222) and receives research funding from Amgen, Bristol Myers Squibb, and Karyopharm. BS is an inventor on United States Provisional Patent Application No.: US20210181179A1 "Diagnosis and treatment of immunotherapyinduced neurotoxicity" filed by Memorial Sloan Kettering Cancer Center, served in a consulting or advisory role for Celgene, Janssen, Legend Biotech, Incyte, and In8bio, and received research funding from ADC Therapeutics. JGP-L served in a consulting or advisory role for Janssen, served in speakers bureau for Eisai, and received research funding from DNAtrix. NWCJvdD received grant support and advisory board fees from Bristol-Myers Squibb/Celgene, Janssen, Novartis, and Amgen, advisory board fees from Takeda, Servier, Bayer, Adaptive Biotechnologies, Roche, GlaxoSmithKline, and Sanofi, and grant support from Cellectis. BA served in a consulting or advisory role for Amgen, Celgene, and Janssen-Cilag, received travel expenses from Amgen, Celgene, Janssen-Cilag, Sanofi, and Takeda, received honoraria from Celgene, Janssen-Cilag, Sanofi, and Takeda, and received research funding from JanssenCilag. M-VM received consulting fees from Janssen-Cilag, GlaxoSmithKline, Celgene, Amgen, Regeneron, Pfizer, AbbVie, Takeda, Roche/Genentech, Sanofi, Oncopeptides, and Adaptive Biotechnologies. DM, KCDB, JMS, HV, LM, EL, IC, NL, YO, EZ, and CL are employees of Janssen. $C \mathrm{CJ}$ is an employee of Janssen and a consultant physician at the Memorial Sloan Kettering Cancer Center (New York, NY, USA). LP, MA, and DG are employees of Legend Biotech. AJ served in a consulting or advisory role for and received honoraria from AbbVie, Amgen, Bristol-Myers Squibb/Celgene, GlaxoSmithKline, Janssen, Juno Therapeutics, Karyopharm Therapeutics, and Sanofi. HE served in a consulting or advisory role for Amgen, Bristol-Myers Squibb, Celgene, Janssen, Novartis, and Takeda, received travel expenses from Amgen, Bristol-Myers Squibb, Celgene, Janssen, and Takeda, received honoraria from Amgen, Bristol-Myers Squibb, Celgene, Janssen, Novartis, and Takeda, and received research funding from Amgen, Bristol-Myers Squibb, Celgene, and Janssen.

\section{ADDITIONAL INFORMATION}

Supplementary information The online version contains supplementary material available at https://doi.org/10.1038/s41408-022-00629-1.

Correspondence and requests for materials should be addressed to Sundar Jagannath.

Reprints and permission information is available at http://www.nature.com/ reprints

Publisher's note Springer Nature remains neutral with regard to jurisdictional claims in published maps and institutional affiliations.

\begin{abstract}
(c) (i)
Open Access This article is licensed under a Creative Commons Attribution 4.0 International License, which permits use, sharing, adaptation, distribution and reproduction in any medium or format, as long as you give appropriate credit to the original author(s) and the source, provide a link to the Creative Commons license, and indicate if changes were made. The images or other third party material in this article are included in the article's Creative Commons license, unless indicated otherwise in a credit line to the material. If material is not included in the article's Creative Commons license and your intended use is not permitted by statutory regulation or exceeds the permitted use, you will need to obtain permission directly from the copyright holder. To view a copy of this license, visit http://creativecommons. org/licenses/by/4.0/.
\end{abstract}

(c) The Author(s) 2022 